\title{
REVISTAS BRASILEIRAS PUBLICADORAS DE ARTIGOS CIENTÍFICOS EM CIRURGIA. I - CARACTERÍSTICAS ESTRUTURAIS E ADMINISTRATIVAS DAS REVISTAS ${ }^{1}$
}

\author{
Dinah Aguiar Población2 \\ Saul Goldenberg3 \\ Edna Frasson de Souza Montero4 \\ Márcia Bento Moreira5 \\ Rosely de Fátima Pellizzon6
}

\section{INTRODUÇÃO}

A expectativa do autor, ao elaborar e publicar o artigo científico, é a de comunicar à comunidade alvo os resultados de suas pesquisas. Com o objetivo de contribuir para o avanço do conhecimento, o pesquisador assume esse compromisso com a sociedade utilizando-se dos meios adequados de comunicação visando alcançar o sucesso esperado. Essa pretensão será atingida, na medida em que o artigo for publicado em revista que seja visível e acessível à comunidade científica. Um dos exemplos é a visibilidade da ciência divulgada pela revista Acta Cirúrgica Brasileira analisada ao longo da trajetória ininterrupta de $15 \operatorname{anos}^{(1)}$. Isto significa que o periódico deve estar inserido no circuito do processo de transferência da informação ${ }^{(2)}$. Nesse fluxo, destacam-se as relações interpessoais e afloram as interações entre grupos e instituições de pesquisa, que influem no processo de comunicação e de produção científica, bem como contribuem para o desenvolvimento global.

Esses temas, explorados em profundidade por vários autores, a partir dos estudos e pesquisas de Price $^{(3)}$ Garvey $^{(4)}$ e Meadows ${ }^{(5)}$, permitem compreen- der as características do processo da produção do conhecimento e ampliam a viabilidade das análises de crescimento da ciência e do estágio em que se encontra cada área de especialidade.

Os pesquisadores, considerando a qualidade da sua contribuição e conscientes de que estão sujeitos à avaliação pelos seus pares, em várias instâncias ${ }^{(6)}$, tornam-se cautelosos na seleção das revistas às quais submeterão os originais para publicação. Parte do sucesso do autor é atribuído às habilidades e competência do editor da revista, uma vez que a ele cabe definir a política editorial $^{(7,8,9)}$, aprimorar a apresentação formal e adotar padrões ou diretrizes recomendadas por organismos internacionais ${ }^{(10-13)}$.

É fácil compreender a posição dos editores que colocam o periódico científico não apenas como o veículo para perpetuar o conhecimento ${ }^{(14-15)}$, mas impulsionados pelos avanços das tecnologias emergentes revertem o dinâmico processo de geração e difusão da informação. Isto significa que as grandes transformações devem ocorrer em virtude da acelerada velocidade imposta aos processos de comunicação, fatos sinalizados por Garfield ${ }^{(16)}$, em visão prospectiva para o século XXI. Para superar os limites dos processos tradicio-

1. Trabalho do Núcleo de Comunicação Científica em Cirurgia (NCCC) da Sociedade Brasileira para o Desenvolvimento da Pesquisa em Cirurgia (SOBRADPEC).

2. Profa. Dra. da Escola de Comunicação e Artes (ECA/USP). Coordenadora do Núcleo de Pesquisa de Produção Científica em Ciência da Informação (NPC/ECA/USP) e do Núcleo de Comunicação Científica em Cirurgia (NCCC) da SOBRADPEC. Pesquisadora I - B do CNPq. E-mail: dinahmap@usp.br

3. Prof. Titular do Depto. de Cirurgia da UNIFESP/EPM. Presidente da SOBRADPEC. Pesquisador I - A CNPq. E-mail: sgolden@terra.com.br

4. Professora Afiliada do Depto. de Cirurgia da UNIFESP/EPM e Pesquisadora do NCCC. E-mail: efsmontero.dcir@epm.br

5. Médica Veterinária, Mestre em Técnica Operatória e Cirurgia Experimental e Pesquisadora do NCCC. E-mail: mazinhabm.dcir@epm.br

6. Bibliotecária Responsável pelo Setor de Referência da Biblioteca Central UNIFESP/EPM e Pesquisadora do NCCC. E-mail: rosely.bc@epm.br 
nais destaca-se o papel da inteligência artificial para a recuperação da informação e as facilidades oferecidas pelo acesso e disponibilidade dos documentos pelas bibliotecas digitais e virtuais.

Seus efeitos benéficos repercutem na comunidade científica e estimulam os autores a sofisticar os meios e métodos de produção dos documentos, objetivando selecionar os veículos de difusão e as revistas de maior impacto, segundo vários autores que vêm se preocupando com as análises bibliométricas e cienciométricas $^{(17,24)}$.

A alta relevância desses estudos, que servem de alerta aos editores médicos, atinge não só aqueles que exercem essa atividade há longos anos, mas oferecem subsídios e constituem instrumental básico para orientar os jovens candidatos, que pretendem iniciar na trilha das atividades concernentes à editoração do periódico científico. De acordo com Kassirer ${ }^{(25)}$ editor do New England Journal of Medicine, ao relatar sua longa experiência, foi constatada a ausência de ensino formal em faculdade ou instituição de ensino superior, para preparar o profissional com a formação adequada resultando na falta de profissionalismo, inviabilizando a criação da carreira. O mesmo ocorre no Brasil onde a publicação de periódico científico é considerado "ato heróico"(26). Para reverter essa situação, algumas iniciativas vêm sendo tomadas durante eventos realizados pela Associação Brasileira de Editores Científicos $(\mathrm{ABEC})^{(27)} \mathrm{e} \mathrm{em}$ nível internacional, a World Association of Medical Editors ${ }^{(28)}$ prepara roteiros, visando conscientizar os editores potenciais a fim de assumirem essas responsabilidades. Com a proposta de contribuir para transformar a "atividade amadorística" do editor e reconhecendo a importância de alertar a comunidade científica para o papel de guardião da qualidade das revistas ${ }^{(29)}$, o Núcleo de Comunicação Científica em Cirurgia (NCCC), vinculado à Sociedade Brasileira para o Desenvolvimento da Pesquisa em Cirurgia (SOBRADPEC) ${ }^{(30)}$ retoma essa temática com a finalidade de oferecer embasamento sólido para estudos e análise das revistas científicas brasileiras onde predominam as especialidades cirúrgicas e a comunicação científica da área.

Abordagens realizadas em anos anteriores mostram a preocupação com diversos aspectos referentes a publicações: 1 - o problema no uso dos descritores nos artigos científicos ${ }^{(31)} ; 2$ - a desigualdade de critérios nas referências bibliográficas ${ }^{(32)} ; 3$ - análise das instruções aos autores publicadas em 19 periódicos médicos $^{(33)} ; 4$ - O periódico como produto: da criação ao autor à inovação do editor ${ }^{(34)}$. 5 - aspectos éticos da pesquisa em seres humanos contidos nas instruções aos autores em revistas científicas brasileiras ${ }^{(35)} ; 6$ - considerações éticas a respeito da publicação do trabalho científico $^{(36)} ; 7$ - normalizar é salutar ${ }^{(37)}$.

Retomando essa linha, o (NCCC) se propõe a apresentar o diagnóstico da situação atual dos processos de comunicação na área, envolvendo as instituições brasileiras responsáveis pela produção do conhecimento. Os esforços despendidos para que a comunidade de pesquisadores brasileiros em cirurgia possa competir no mesmo nível dos pesquisadores internacionais, representam um desafio só enfrentado por instituições fortes, com recursos humanos qualificados que utilizam adequadamente os processos de comunicação e os veículos tradicionais e eletrônicos ${ }^{(34,38-40)}$ de difusão do conhecimento.

Nessa linha de pesquisa, centrada no processo de comunicação científica entre pesquisadores da área da saúde e análise dos fluxos de informação que permitem as relações sociais entre as comunidades científicas, foi estabelecida a primeira abordagem privilegiando o periódico científico.

Partindo-se da análise desse veículo, torna-se viável identificar as suas características, interpretar o fluxo de comunicação e interação entre os pesquisadores, grupos e instituições de pesquisa e a projeção da comunidade brasileira da área cirúrgica nos meios internacionais.

Para estudar a produção do conhecimento da área cirúrgica publicada no Brasil, compreender os padrões de coerência dos autores e dos editores dos periódicos, cujos indicadores de qualidade ${ }^{(41-43)}$ foram considerados nas análises dos artigos cirúrgicos, nesta linha de investigação foram planejadas etapas com a seguinte seqüência:

I - Características estruturais e administrativas das revistas científicas brasileiras que publicam artigos cirúrgicos.

II - Terminologia adotada pelo editor para designar os membros do corpo editorial e atribuições pertinentes aos componentes da equipe responsável pela política, administração e qualidade do conteúdo da revista.

III - Instruções aos autores relacionada com a observância na publicação.

IV - Identificação do perfil dos autores.

V - Competitividade da produção nacional com a internacional diagnosticando o fator de impacto.

Fundamentado na importância de avaliar a qualidade das revistas brasileiras da área médica que publicam artigos da especialidade cirúrgica, reconhecendo que o êxito da difusão da informação é decorrente do processo de transferência do conhecimento segundo normas e padrões reconhecidos internacionalmente, a 
primeira etapa desta linha de pesquisa tem por finalidade delimitar e caracterizar as revistas brasileiras que fazem parte do universo da investigação.

\section{OBJETIVOS}

- Selecionar as revistas científicas brasileiras que publicam artigos cirúrgicos de acordo com a análise de conteúdo.

- Analisar as características estruturais apresentadas pelas revistas baseando-se em padrões internacionais relacionados com elaboração e apresentação de publicações periódicas.

- Identificar os indicadores de qualidade que os responsáveis pela editoração das revistas selecionadas investiram nas publicações para garantir o êxito do fluxo de transferência da informação.

\section{MÉTODOS}

A pesquisa documental iniciada a partir da Base LILACS (Literatura Latino-Americana e do Caribe em Ciências da Saúde) produzida pela BIREME permitiu identificar as revistas brasileiras que publicam artigos de diferentes especialidades da área. Atualmente, compõem essa Base 695 títulos de periódicos da América Latina dos quais 246 (35\%) são publicados no Brasil. Considerando-se somente as revistas brasileiras que publicam artigos científicos na área cirúrgica, classificados como originais ou de revisão, foi delimitado o universo de acordo com os seguintes critérios:

\section{Universo da investigação}

Critérios para exclusão:

- Relacionados ao título da revista, identificado na base LILACS:

- Boletim, Informativo, Noticiário, Revista da Faculdade... Revista da Fundação...do Hospital... do Instituto... (Endogenia institucional)

Revista Médica... Revista de Medicina (âmbito genérico induzindo a artigos clínicos)

- Relacionados com a periodicidade, identificada na fonte primária:

- Irregularidade na publicação da revista de acordo com a periodicidade mencionada nos últimos cinco anos

- Dificuldade de acesso ao fascículo mais recente publicado no ano 2001 e alguns fascículos do período delimitado (1998-2000), decorrente da localização em bibliotecas brasileiras.
Critérios para inclusão:

- As revistas da área de cirurgia foram utilizadas como fontes primárias e as que contemplavam os requisitos exigidos quanto ao título, periodicidade e acesso foram submetidas à análise de conteúdo dos artigos publicados nos volumes completos dos três últimos anos (1998 a 2000).

- As revistas não disponíveis no acervo da BIREME foram obtidas em várias bibliotecas da área da saúde em diferentes estados. Como estratégias para completar a fase de coleta dos documentos, não obtidos nas bibliotecas, foram realizadas gestões junto aos editores de algumas revistas.

\section{Caracterização do conteúdo das revistas selecionadas}

- Para análise temática os artigos foram categorizados por profissionais da área médica, em:

- cirúrgicos (foram selecionadas as revistas que publicaram $10 \%$ ou mais de artigos cirúrgicos no triênio)

- não cirúrgicos (percentual dos artigos que completam o conteúdo das revistas selecionadas para compor o universo da pesquisa)

- outros: equipamentos, produtos, legislação, resumos de comunicações em congressos, editoriais etc... (análise idêntica aos artigos não cirúrgicos)

- Desta análise temática foram selecionados somente artigos cirúrgicos, focalizados por subcategorias quanto a:

- artigos originais

- artigos de revisão (incluindo atualização e trabalhos especiais)

- Os artigos originais foram abordados sob os seguintes aspectos:

- estudos em seres humanos

- estudos em animais de experimentação

\section{Quantificação dos artigos cirúrgicos quanto a paginação}

- Análise quantitativa da distribuição da matéria de cada uma das publicações (1998 a 2000)

- total de páginas por ano de cada revista

- total de páginas cirúrgicas por ano de cada revista

- total de artigos publicados em cada ano 
Características dos processos de editoração e difusão da publicação

Para avaliação de alguns preceitos, segundo os parâmetros determinados por organismos e entidades internacionais constantes dos manuais de estilo(44-47), relacionados com o processo de editoração de publicações científicas, os dados foram extraídos do último fascículo publicado no ano 2001. Os aspectos gerais e itens apresentados pela própria revista foram considerados na análise, desde que constituem fatores reconhecidamente determinantes da garantia de sucesso e consistência do ciclo de transferência da informação.

Os resultados são apresentados em forma de tabelas e gráficos e oferecem subsídios iniciais para o diagnóstico da contribuição dos autores que publicam artigos cirúrgicos em revistas científicas brasileiras.

\section{RESULTADOS}

\section{I - Caracterização do conteúdo das revistas selecionadas}

Das 30 revistas identificadas com as credenciais necessárias para inclusão, dentro dos critérios relacionados ao título, periodicidade e acesso, somente 23 apresentavam $10 \%$ ou mais de artigos cirúrgicos após análise de conteúdo dos volumes completos publicados no período delimitado (1998 a 2000)(Tabela 1).

TABELA 1 - Distribuição das revistas brasileiras selecionadas publicadoras de artigos segundo a categoria cirurgia*, com as subcategorias dos artigos de revisão e originais. Artigos originais sob os aspectos de estudos em seres humanos ou em animais de experimentação (19982000).

\begin{tabular}{|c|c|c|c|c|c|c|c|c|c|c|c|}
\hline \multirow{4}{*}{ Títulos } & \multirow{4}{*}{ Total de artigos } & \multicolumn{10}{|c|}{ Artigos Cirúrgicos } \\
\hline & & \multirow{2}{*}{\multicolumn{2}{|c|}{ Total }} & \multirow{2}{*}{\multicolumn{2}{|c|}{ Revisão }} & \multicolumn{6}{|c|}{ Originais } \\
\hline & & & & & & \multicolumn{2}{|c|}{ Humanos } & \multicolumn{2}{|c|}{ Animais } & \multicolumn{2}{|c|}{ Total } \\
\hline & & $\mathbf{N}$ & $\%$ & $\mathbf{N}$ & $\%$ & $\mathbf{N}$ & $\%$ & $\mathbf{N}$ & $\%$ & $\mathbf{N}$ & $\%$ \\
\hline Acta Cir Bras & 125 & 101 & 81 & 8 & 7 & 8 & 8 & 85 & 84 & 93 & 92 \\
\hline Acta Ortop Bras & 69 & 46 & 67 & 4 & 7 & 38 & 83 & 4 & 9 & 42 & 91 \\
\hline An Paul Med Cir & 62 & 38 & 61 & 10 & 18 & 28 & 74 & 0 & 0 & 28 & 74 \\
\hline Arq Bras Cardiol & 431 & 87 & 20 & 14 & 14 & 66 & 76 & 7 & 8 & 73 & 84 \\
\hline Arq Bras Neurocir & 81 & 44 & 54 & 6 & 11 & 38 & 86 & 0 & 0 & 38 & 86 \\
\hline Arq Bras Oftalmol & 279 & 63 & 23 & 7 & 10 & 52 & 83 & 4 & 6 & 56 & 89 \\
\hline Arq Gastroenterol & 120 & 21 & 18 & 1 & 4 & 17 & 81 & 3 & 14 & 20 & 95 \\
\hline Braz J Urol & 262 & 101 & 39 & 13 & 12 & 80 & 79 & 8 & 8 & 88 & 87 \\
\hline GED Gastroenterol Endosc Diag & 159 & 35 & 22 & 9 & 17 & 25 & 71 & 1 & 3 & 26 & 74 \\
\hline $\mathrm{J}$ Bras Neurocir & 66 & 19 & 29 & 0 & 0 & 19 & 100 & 0 & 0 & 19 & 100 \\
\hline J Pneumol & 172 & 28 & 16 & 3 & 8 & 19 & 68 & 6 & 21 & 25 & 89 \\
\hline Rev Assoc Med Bras & 179 & 42 & 23 & 9 & 16 & 27 & 64 & 6 & 14 & 33 & 79 \\
\hline Rev Bras Anestesiol & 195 & 71 & 36 & 10 & 12 & 60 & 85 & 1 & 1 & 61 & 86 \\
\hline Rev Bras Cir Cardiovasc & 139 & 129 & 93 & 2 & 2 & 117 & 91 & 10 & 8 & 127 & 98 \\
\hline Rev Bras Coloproctol & 139 & 70 & 50 & 13 & 15 & 56 & 80 & 1 & 1 & 57 & 81 \\
\hline Rev Bras Ginecol Obstet & 219 & 57 & 26 & 0 & 0 & 53 & 93 & 4 & 7 & 57 & 100 \\
\hline Rev Bras Mastologia & 101 & 32 & 32 & 11 & 21 & 16 & 50 & 5 & 16 & 21 & 66 \\
\hline Rev Bras Oftalmol & 347 & 110 & 32 & 26 & 20 & 82 & 75 & 2 & 2 & 84 & 76 \\
\hline Rev Bras Ortop & 371 & 252 & 68 & 12 & 5 & 230 & 91 & 10 & 4 & 240 & 95 \\
\hline Rev Bras Otorrinolaringol & 224 & 89 & 40 & 3 & 3 & 86 & 97 & 0 & 0 & 86 & 97 \\
\hline Rev Col Bras Cir & 230 & 172 & 75 & 5 & 3 & 143 & 83 & 24 & 14 & 167 & 97 \\
\hline Rev Ginec Obstet & 125 & 21 & 17 & 1 & 4 & 20 & 95 & 0 & 0 & 20 & 95 \\
\hline Sao Paulo Med J & 146 & 36 & 25 & 0 & 0 & 36 & 100 & 0 & 0 & 36 & 100 \\
\hline TOTAL & 4241 & 1664 & 39 & 167 & 10 & 1316 & 88 & 181 & 12 & 1497 & 90 \\
\hline
\end{tabular}

*Revistas brasileiras que publicaram no triênio $10 \%$ ou mais de artigos de cirurgia. 
As 23 revistas selecionadas publicaram 4241 artigos que foram categorizados:

$\begin{array}{lrlr}\text { outros } & 42 & - & 1 \% \\ \text { cirúrgicos } & 1664 & - & 39 \% \\ \text { não cirúrgicos } & 2535 & - & 60 \%\end{array}$

Das 23 revistas 8 (4\%) publicaram no período $50 \%$ ou mais dos 1664 artigos cirúrgicos (originais e revisão) (Figura 1):

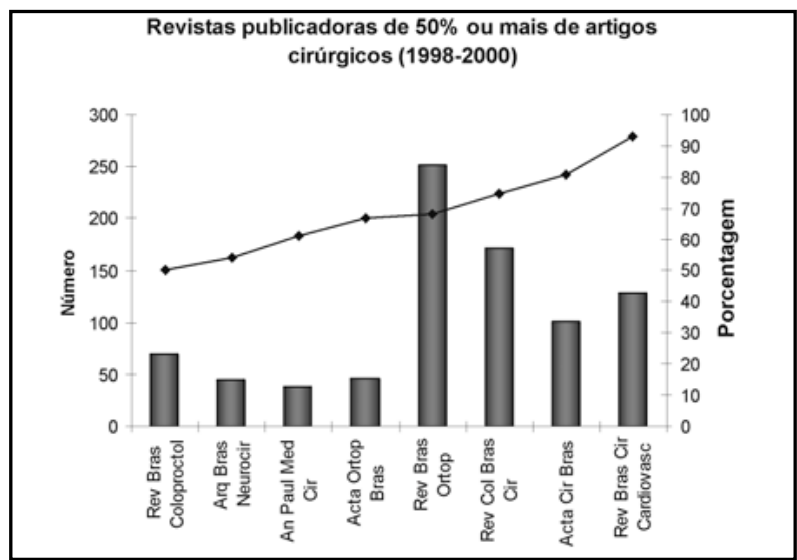

FIGURA 1 - Gráfico que mostra a porcentagem crescente das revistas publicadoras de $50 \%$ ou mais de artigos cirúrgicos (1998-2000).

A distribuição dos 1664 artigos cirúrgicos publicados no triênio, foi selecionada segundo os enfoques de revisão 167 (10\%) e originais 1497 (90\%).

Nesta primeira fase da investigação não são considerados os artigos de revisão, que representam 10\% do total de artigos cirúrgicos. Estes indicadores quantitativos serão combinados com a análise das características do artigo de revisão por ocasião da interpretação das instruções apresentadas aos autores pelos editores.

Dos 1497 artigos cirúrgicos originais 1316 (88\%) referem-se a seres humanos e $181(12 \%)$ a animais de experimentação. Dos 1316 artigos originais categorizados como cirúrgicos em humanos, 967 (74\%) predominam em 14 periódicos, onde estão representados por 80 a $100 \%$ do conteúdo dessas publicações editadas no triênio. (Figura 2).

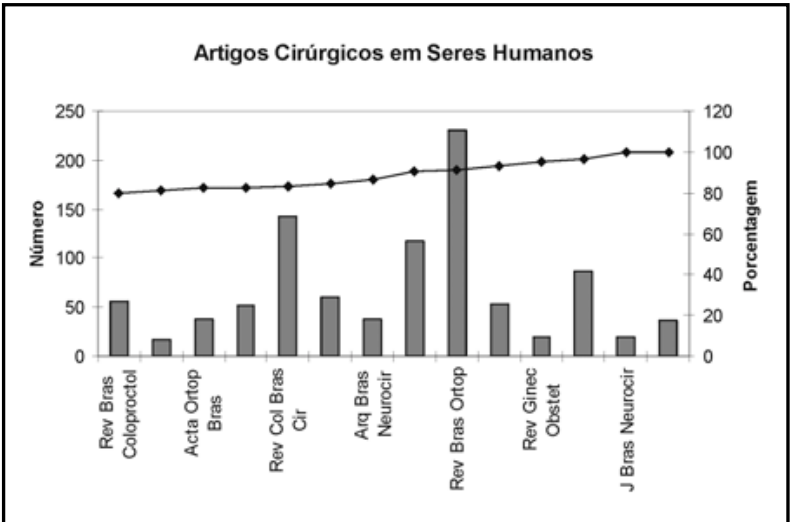

FIGURA 2 - Gráfico que mostra a porcentagem crescente dos artigos cirúrgicos originais, segundo o aspecto de estudo em seres humanos.
Os artigos originais categorizados como cirúrgicos em animais (181) estão representados em 17 periódicos, com uma variabilidade muito grande ( $1 \%$ a $84 \%)$ (Figura 3).

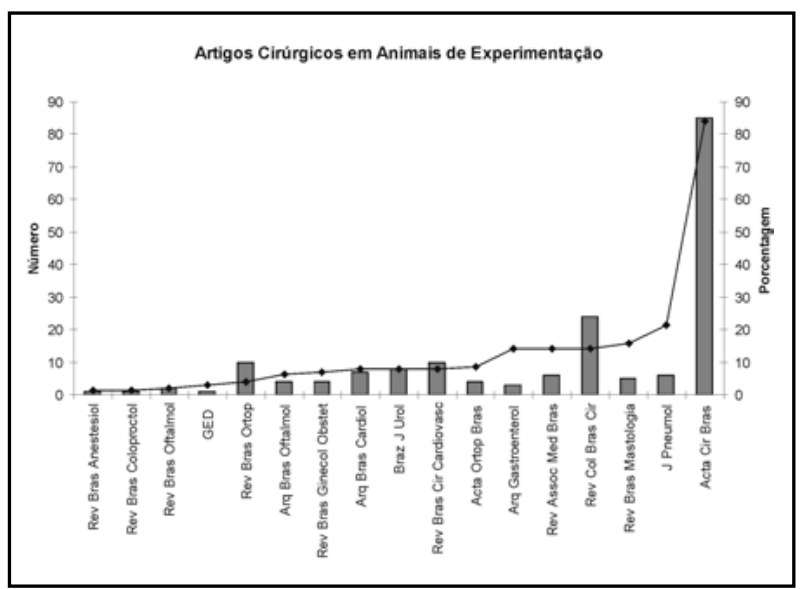

FIGURA 3 - Gráfico que mostra a porcentagem crescente dos artigos cirúrgicos originais, segundo o aspecto de estudo em animais de experimentação.

\section{II - Quantificação dos artigos cirúrgicos quanto à paginação}

Os 72 volumes analisados correspondem a 28110 páginas publicadas no triênio, das quais 8810 (31\%) foram dedicadas aos artigos cirúrgicos (Tabela 2 Figura 4). Observa-se também, que a média do número de páginas cirúrgicas é de 2937, com tendência a queda no triênio. 
TABELA 2 - Distribuição das revistas brasileiras selecionadas segundo os fascículos de cada volume e o total de páginas publicadas em cada ano do triênio, identificando o total das páginas dos artigos cirúrgicos (1998-2000).

\begin{tabular}{|c|c|c|c|c|c|c|c|c|c|c|}
\hline \multirow{4}{*}{ TÍTULOS } & \multirow[b]{3}{*}{ Volumes e Números } & \multicolumn{8}{|c|}{ Total de Páginas } & \multirow[b]{3}{*}{2000} \\
\hline & & \multirow[t]{3}{*}{1998} & \multirow[t]{3}{*}{1999} & \multirow[t]{3}{*}{2000} & \multirow[t]{3}{*}{ Triênio } & \multicolumn{5}{|c|}{ Páginas Cirúrgicas } \\
\hline & & & & & & \multicolumn{2}{|c|}{ Triênio } & \multirow[t]{2}{*}{1998} & \multirow[t]{2}{*}{1999} & \\
\hline & & & & & & $\mathbf{N}$ & $\%$ & & & \\
\hline Acta Cir Bras & \multirow{2}{*}{$\begin{array}{c}13(1-4), 14(1-4) \text { e } 15(1-4) \\
6(1-4), 7(1-4) \text { e } 8(1-4)\end{array}$} & 296 & 232 & 254 & 782 & 605 & 77 & 233 & 175 & 197 \\
\hline Acta Ortop Bras & & 188 & 188 & 208 & 584 & 391 & 67 & 71 & 147 & 173 \\
\hline An Paul Med Cir & $125(1-4), 126(1-4)$ e $127(1-4)$ & 148 & 136 & 288 & 572 & 249 & 44 & 65 & 90 & 94 \\
\hline Arq Bras Cardiol & $\begin{array}{c}70(1-6), 71(1-6) \\
72(1-6),(1-6) \text { e } 75(1-6)\end{array}$ & 883 & 761 & 1104 & 2748 & 317 & 12 & 134 & 47 & 136 \\
\hline Arq Bras Neurocir & $17(1-4), 18(1-4)$ e $19(1-3)$ & 172 & 232 & 156 & 560 & 286 & 51 & 75 & 138 & 73 \\
\hline Arq Bras Oftalmol & $61(1-6), 62(1-6)$ e $63(1-6)$ & 736 & 776 & 532 & 2044 & 289 & 14 & 123 & 104 & 62 \\
\hline Arq Gastroenterol & $35(1-4), 36(1-4)$ e $37(1-4)$ & & 276 & 52 & 637 & & & 15 & 68 & 39 \\
\hline Braz J Urol & $24(1-6), 25(1-6)$ e $26(1-6)$ & 337 & 566 & 663 & 1566 & 51 & 33 & 135 & 205 & 172 \\
\hline GED & $17(1-6), 18(1-6)$ e $19(1-6)$ & 232 & 270 & 248 & 750 & 144 & 19 & 37 & 75 & 32 \\
\hline J Bras Neurocir & $9(1-3), 10(1-3)$ e $11(1-3)$ & 116 & 114 & 113 & 343 & 122 & 36 & 40 & 25 & 57 \\
\hline J Pneumol & $24(1-6), 25(1-6)$ e $26(1-6)$ & 388 & 356 & 354 & 1098 & 108 & 10 & 16 & 36 & 56 \\
\hline Rev Assoc Med B & $44(1-4), 45(1-4)$ e $46(1-4)$ & 352 & & 36 & 1100 & 23 & 21 & 85 & 64 & 84 \\
\hline Rev Bras Anestesiol & $48(1-6), 49(1-6)$ e $50(1-6)$ & 620 & 464 & 528 & 1612 & 38 & 24 & 113 & 145 & 125 \\
\hline $\begin{array}{l}\text { Rev Bras Cir } \\
\text { Cardiovasc }\end{array}$ & $13(1-4), 14(1-4)$ e $15(1-4)$ & 396 & 374 & 358 & 1128 & 932 & 83 & 330 & 295 & 307 \\
\hline Rev Bras Coloproctol & $18(1-4), 19(1-4)$ e $20(1-4)$ & 290 & 320 & 278 & 888 & 360 & 41 & 126 & 121 & 113 \\
\hline $\begin{array}{c}\text { Rev Bras Ginecol } \\
\text { Obstet }\end{array}$ & $20(1-10), 21(2-3,5-10)$ e $22(1-10)$ & 607 & 635 & 621 & 1863 & 293 & 16 & 104 & 92 & 97 \\
\hline Rev Bras Mastologia & $8(1-4), 9(1-4)$ e $10(1-4)$ & 221 & & 218 & 617 & 104 & 17 & 33 & 24 & 47 \\
\hline Rev Bras Oftalmol & $57(1-12), 58(1-12)$ e $59(1-12)$ & 799 & 81 & 920 & 2534 & 472 & 19 & 155 & 206 & 111 \\
\hline Rev Bras Ortop & $33(1-12), 34(1-12)$ e $35(1-12)$ & 1020 & 598 & 468 & 2086 & 1341 & 64 & 714 & 368 & 259 \\
\hline Rev Bras & $64(1-6), 65(1-6)$ e $66(1-6)$ & 513 & 552 & 708 & 1773 & 457 & 26 & 98 & 161 & 198 \\
\hline - 1 & & & & & & & & & & \\
\hline Rev Col Bras Cir & $25(1-6), 26(1-6)$ e $27(1-6)$ & 446 & 406 & 442 & 1294 & 851 & 66 & 284 & 227 & 340 \\
\hline Rev Ginec Obstet & $9(1-4), 10(1-4)$ e $11(1-4)$ & 273 & 238 & 258 & 769 & 79 & 10 & 25 & 25 & 29 \\
\hline Sao Paulo Med J & $116(1-6), 117(1-6)$ e $118(1-6)$ & 283 & 276 & 203 & 762 & 160 & 21 & 72 & 62 & 26 \\
\hline TOTAL & Números=408 & 9625 & 9143 & 9342 & 28110 & 8810 & 31 & 3083 & 2900 & 2827 \\
\hline
\end{tabular}

Comparando-se as revistas que contemplaram as maiores porcentagens $(41-83 \%)$ de páginas dedicadas aos artigos cirúrgicos, verifica-se que coincidem com

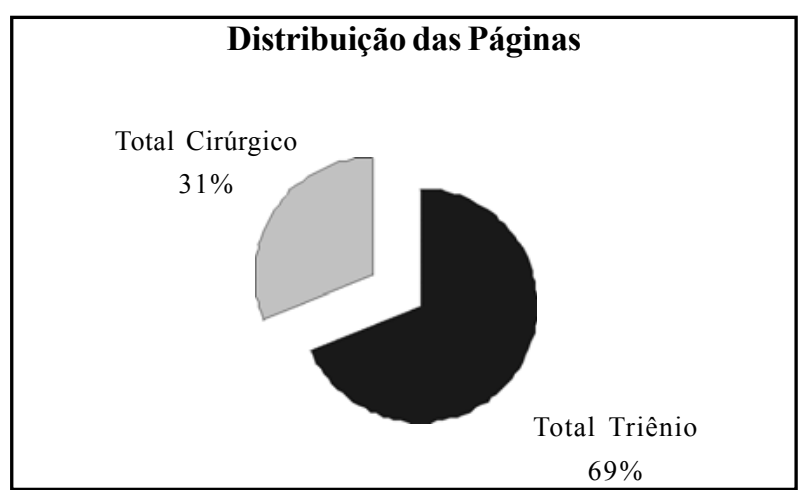

FIGURA 4 - Mostra a quantificação das páginas cirúrgicas em relação ao total de páginas publicadas no triênio (1998-2000).

\section{III - Características dos processos de editoração e difusão da publicação.}

A avaliação das revistas, analisadas a partir dos dados do último volume publicado em 2001, foi trabalhada em relação aos seguintes aspectos:

> Características da idade da publicação, entidade responsável e periodicidade. as revistas que publicaram $50 \%$ ou mais de artigos cirúrgicos. (Figuras 4 e 5)

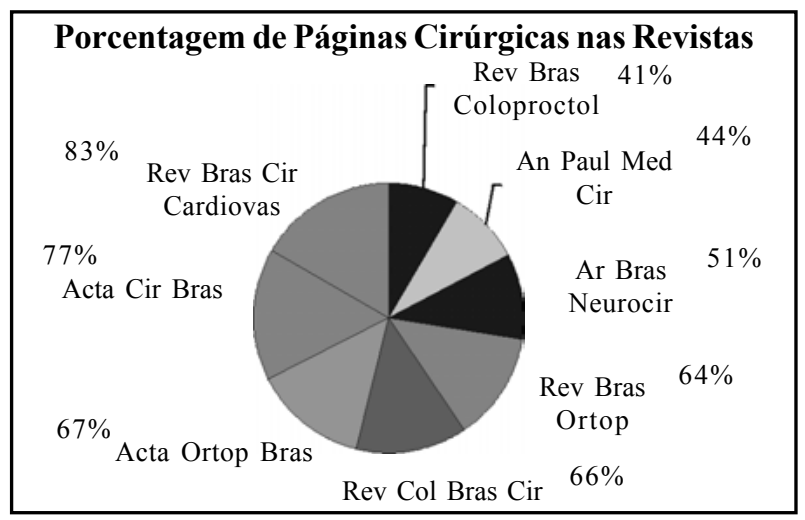

FIGURA 5 - Mostra a porcentagem de páginas cirúrgicas em relação às revistas selecionadas.

> Características da difusão e visibilidade para a comunidade científica.

\section{Idade da publicação, entidade responsável e periodicidade}

A Tabela 3 apresenta os 23 títulos selecionados com data inicial do volume 1 e o último volume publicado no ano de 2001. 
TABELA 3 - Distribuição das revistas brasileiras selecionadas publicadoras de artigos segundo as características estruturais e administrativas dos processos de editoração (1998-2000).

\begin{tabular}{|c|c|c|c|c|c|}
\hline TÍTULOS & $\begin{array}{l}\text { DATA } \\
\text { Vol } 1 \\
\end{array}$ & $\begin{array}{c}\text { Volume } \\
2001 \text { último } \\
\end{array}$ & Periodicidade & ISSN & $\begin{array}{c}\text { Entidade } \\
\text { Responsável } \\
\end{array}$ \\
\hline Acta Cir Bras & 1986 & 16 & Trimestral & $0102-8650$ & ${ }^{1}$ SOBRADPEC \\
\hline Acta Ortop Bras & 1993 & 9 & Trimestral & $1413-7852$ & ${ }^{2}$ DOT APM/SBOT-Reg. SP \\
\hline An Paul Med Cir & 1913 & 128 & Trimestral & $0003-245 X$ & Real e Ben. Soc. Port. de SP \\
\hline Arq Bras Cardiol & 1948 & $77-78$ & Mensal & $0066-782 X$ & Soc. Bras. de Cardiologia \\
\hline Arq Bras Neurocir & 1982 & 20 & Trimestral & $0103-5355$ & Soc. Bras. de Neurocirurgia \\
\hline Arq Bras Oftalmol & 1938 & 64 & Bimestral & 0004-2749 & Cons. Bras. de Oftalmologia \\
\hline Arq Gastroenterol & 1964 & 38 & Trimestral & 0004-2803 & ${ }^{3}$ IBEPEGE \\
\hline * Braz J Urol & 1975 & 27 & Bimestral & $1517-6878$ & Soc. Bras. de Urologia \\
\hline GED & 1982 & 20 & Bimestral & 0101-7772 & ${ }^{4}$ SOBED / FBG / SBH \\
\hline J Bras Neurocir & 1989 & 12 & Quadrimestral & 0103-5118 & Acad. Bras. de Neurocirurgia \\
\hline J Pneumol & 1975 & 27 & Bimestral & $0102-3586$ & ${ }^{5} \mathrm{SBPT}$ \\
\hline Rev Assoc Med Bras & 1992 & 47 & Bimestral & $0104-4230$ & Associação Médica Brasileira \\
\hline Rev Bras Anestesiol & 1951 & 28 & Bimestral & 0034-7094 & Soc. Bras. de Anestesiologia \\
\hline Rev Bras Cir Cardiovasc & 1986 & 16 & Trimestral & $0102-7638$ & $\begin{array}{l}\text { Soc. Bras. de Cir. } \\
\text { Cardiovascular }\end{array}$ \\
\hline Rev Bras Coloproctol & 1981 & 21 & Trimestral & 0101-9880 & Soc. Bras. de Coloproctologia \\
\hline Rev Bras Ginecol Obstet & 1979 & 23 & Mensal & $0100-7203$ & ${ }^{6}$ FEBRASGO \\
\hline Rev Bras Mastologia & 1991 & 21 & Trimestral & 0104-8058 & ${ }^{7} \mathrm{SBM} / \mathrm{AMB}$. \\
\hline Rev Bras Oftalmol & 1942 & 60 & Mensal & $0034-7280$ & Soc. Bras. de Oftalmologia \\
\hline Rev Bras Ortop & 1966 & 36 & Mensal & $0102-3616$ & ${ }^{8} \mathrm{SBOT}$ \\
\hline Rev Bras Otorrinolaringol & 1939 & 67 & Bimestral & 0034-7299 & $\begin{array}{l}\text { Soc. Bras. de } \\
\text { Otorrinolaringologia }\end{array}$ \\
\hline Rev Col Bras Cir & 1974 & 28 & Bimestral & 0100-6991 & $\begin{array}{l}\text { Colégio Brasileiro } \\
\text { de Cirurgiões }\end{array}$ \\
\hline Rev Ginec Obstet & 1992 & 12 & Trimestral & 0103-7714 & $\begin{array}{l}\text { Inst. da Mulher do } \\
\text { HC-FMUSP }\end{array}$ \\
\hline **São Paulo Med J & 1941 & 119 & Bimestral & $1516-3180$ & $\begin{array}{l}\text { Associação Paulista } \\
\text { de Medicina }\end{array}$ \\
\hline
\end{tabular}

* De 1975 v.1 - até 1999 v. 25 - J Bras Urol - ISSN 0100-0519

** De 1941 v.1 - até 1993 v. 111 - Rev Paul Med - ISSN 0035-0362

1 Sociedade Brasileira para o Desenvolvimento da Pesquisa em Cirurgia - SOBRADPEC

2 Depto. de Ortopedia e Traumatologia da Associação Paulista e Medicina e Sociedade Brasileira de Ortopedia / Traumatologia Regional de São Paulo - DOT APM/SBOT-Reg. SP

3 Instituto Brasileiro de Estudos e Pesquisas de Gastroenterologia - IBEPEGE

4 Sociedade Brasileira de Endoscopia Digestiva - SOBED / Federação Brasileira de Gastroenterologia - FBG / Sociedade Brasileira de Hepatologia - SBH

5 Sociedade Brasileira de Pneumologia e Tisiologia - SBPT

6 Federação Brasileira das Sociedades de Ginecologia e Obstetrícia - FEBRASGO

7 Sociedade Brasileira de Mastologia/Depto. Científico da Associação Médica Brasileira-SBM/AMB

8 Sociedade Brasileira de Ortopedia / Traumatologia Regional de São Paulo - DOT APM/SBOT

O período de existência ininterrupta que varia de 10 anos (1993) a 90 anos (1913) comprova que estas revistas estão consolidadas, atravessam o Rubicon e caminham para uma longevidade garantida pelo suporte de instituições fortes como são as academias, associações, colégios, sociedade e instituições de pesquisa. Essas entidades obedecem aos preceitos de normalização quanto ao registro de ISSN - International Standard Serial Number. Constatado em $100 \%$ das revistas. Dentro da política explicitada na publicação, elas vêm mantendo a periodicidade estipulada, que varia no conjunto: mensal(4); bimestral(9); trimenstral(9) e quadrimestral(1).

\section{Difusão e visibilidade (Recursos e indexação, tiragem, distribuição)}

Esses indicadores bastante significativos de credibilidade são comparados na tabela 4, pela difusão da informação primária por meio de tiragem que varia de 1000 a 15000 exemplares. Esse dado não foi encontrado em 14 (61\%) periódicos publicados em 2001. A distribuição, que é um dos requisitos para a visibilidade da comunidade científica, mostra que é destinada gratuitamente aos sócios (8) das entidades publicadoras; por assinatura (9); combinando distribuição aos sócios e assinatura (5); distribuição sócios e permuta com instituições (3); enquanto 5 não mencionam o processo de distribuição. 
TABELA 4 - Distribuição das revistas brasileiras selecionadas publicadoras de artigos segundo as características estruturais e administrativas dos processos de difusão e visibilidade (1998-2000).

\begin{tabular}{|c|c|c|c|c|c|}
\hline TÍTULOS & Tiragem & Distribuição [Circulação] & Propaganda & Apoio Financeiro & $\begin{array}{l}\text { Acesso* - Indexação } \\
\text { em Fontes secundárias }\end{array}$ \\
\hline $\begin{array}{l}\text { Acta Cir Bras } \\
\text { http://www.scielo.br }\end{array}$ & 1000 & Sócios / permuta & não apresenta & $\mathrm{CNPq}$ & $\begin{array}{l}\text { LILACS / SciELO / } \\
\text { Free Medical Journal }\end{array}$ \\
\hline Acta Ortop Bras & não consta & não consta & capas & não consta & LILACS \\
\hline An Paul Med Cir & não consta & Assinatura & capas / meio dos artigos & não consta & LICACS \\
\hline $\begin{array}{l}\text { Arq Bras Cardiol } \\
\text { http://www.scielo.br }\end{array}$ & 8000 & Assinatura & capas / meio dos artigos & não consta & $\begin{array}{l}\text { MEDLINE/LILACS/ } \\
\text { EMBASE/ SciELO }\end{array}$ \\
\hline $\begin{array}{l}\text { Arq Bras Neurocir } \\
\text { http://www.sbn.com.br }\end{array}$ & não consta & Assinatura & capas / meio dos artigos & não consta & LILACS \\
\hline $\begin{array}{l}\text { Arq Bras Oftalmol } \\
\text { http://www.scielo.br (2002) }\end{array}$ & 6500 & não consta & capas / meio dos artigos & não consta & $\begin{array}{l}\text { LILACS / Free Medical } \\
\text { Journal / SciELO }\end{array}$ \\
\hline $\begin{array}{l}\text { Arq Gastroenterol } \\
\text { http://www.scielo.br }\end{array}$ & não consta & Assinatura & capas / meio dos artigos & não consta & $\begin{array}{l}\text { MEDLINE/ LILACS/ } \\
\text { EMBASE/ SciELO/ CAB } \\
\text { CAB ABS. TROP. DIS. } \\
\text { BULL/ PERIODICA }\end{array}$ \\
\hline $\begin{array}{l}\text { Braz J Urol } \\
\text { http://www.brazjurol. com.br }\end{array}$ & 6000 & Assinatura & capas / meio dos artigos & MCT & EMBASE / LILACS \\
\hline $\begin{array}{l}\text { GED Gastroenterol } \\
\text { Endosc Diag }\end{array}$ & não consta & Sócios / Assinatura & capas / meio dos artigos & não consta & LILACS/ EMBASE \\
\hline $\begin{array}{l}\text { J Bras Neurocir } \\
\text { Endosc Diag }\end{array}$ & não consta & não consta & capas & não consta & LILACS \\
\hline $\begin{array}{l}\text { J Pneumol } \\
\text { http://www.scielo.br (2002) }\end{array}$ & não consta & Assinatura & capas / meio dos artigos & CNPq/ MCT/ FINEP & $\begin{array}{l}\text { LILACS/ PERIODICA / } \\
\text { SciELO }\end{array}$ \\
\hline $\begin{array}{l}\text { Rev Assoc Med Bras } \\
\text { http://www.scielo.br }\end{array}$ & não consta & Sócios / Assinatura & capas / meio dos artigos & não consta & $\begin{array}{l}\text { LILACS / CHEMICAL } \\
\text { ABSTRACTS / SciELO }\end{array}$ \\
\hline Rev Bras Anestesiol & não consta & não consta & capas / pág. prefaciais & não consta & LILACS/ EMBASE \\
\hline $\begin{array}{l}\text { Rev Bras Cir Cardiovasc } \\
\text { http://www.scielo.br }\end{array}$ & não consta & Sócios & capas & CNPq/ FAPESP & LILACS/ SciELO \\
\hline $\begin{array}{l}\text { Rev Bras Coloproctol } \\
\text { http://www.sbcp. org.br }\end{array}$ & não consta & Assinatura / Inst. & capas / pág. prefaciais & não consta & LILACS \\
\hline $\begin{array}{l}\text { Rev Bras Ginec Obstet } \\
\text { http://www.scielo.br (2002) }\end{array}$ & 15000 & Sócios / assinatura & capas / meio dos artigos & não consta & LILACS / SciELO \\
\hline $\begin{array}{l}\text { Rev Bras Mastologia } \\
\text { http://www.lemos. com.br }\end{array}$ & 3000 & Assinatura & capas / meio dos artigos & não consta & LILACS \\
\hline Rev Bras Oftalmol & 5000 & Assinatura & capas / meio dos artigos & não consta & LILACS \\
\hline Rev Bras Ortop & não consta & Assinatura / Sócios & capas / pág. prefaciais & não consta & $\begin{array}{l}\text { LILACS/ EMBASE/ } \\
\text { BIOL. ABSTRACTS }\end{array}$ \\
\hline $\begin{array}{l}\text { Rev Bras Otorrinolaringol } \\
\text { http://www.scielo.br }\end{array}$ & 5500 & Sócios & capas / meio dos artigos & não consta & $\begin{array}{l}\text { EMBASE / LILACS / } \\
\text { SciELO }\end{array}$ \\
\hline $\begin{array}{l}\text { Rev Col Bras Cir } \\
\text { http://www.cbc.org.br }\end{array}$ & 6000 & Sócios/Inst./Assinatura & capas & não consta & LILACS \\
\hline Rev Ginec Obstet & não consta & não consta & capas / meio dos artigos & não consta & LILACS \\
\hline $\begin{array}{l}\text { São Paulo Med J } \\
\text { http://www.scielo.br }\end{array}$ & não consta & Assinatura & capas & não consta & $\begin{array}{l}\text { MEDLINE / LILACS / } \\
\text { SciELO }\end{array}$ \\
\hline \multicolumn{6}{|c|}{$\begin{array}{l}\text { * Acesso: } \\
\text { LILACS - http://www.bireme.br } \\
\text { SciELO - http://www.bireme.br } \\
\text { Free Medical Journal - http://www.freemedicaljournals.com } \\
\text { MEDLINE - http://www.bireme.br } \\
\text { EMBASE - http://www.embase.com } \\
\text { CAB Abstracts - www.cabi-publishing.org/ } \\
\text { PERIÓDICA - http://www.dgbiblio.unam.mx/periodica.html } \\
\text { CAB Abstracts Tropical Diseases Bulletin- http://www.cabi-publishing.org/focus/tropical_diseases/Index.asp } \\
\text { Biological Abstracts - http://www.biosis.org/products_services/ba.html }\end{array}$} \\
\hline
\end{tabular}

A captação de recursos para manter a publicação foi quase exclusivamente através das propagandas impressas nas capas e no meio dos artigos. A única exceção é a Acta Cicúrgica Brasileira que não apresenta propaganda. A realidade desta análise mostra que apenas $4(17 \%)$ das revistas informam ter apoio e subvenção governamental parcial (Acta Cirúrgica Brasileira, Brazilian Journal of Urology, Jornal de Pneumologia e Revista Brasileira de Cirurgia Cardiovascular).

Quanto às fontes de informação secundárias representadas pelas Bases Latino-americanas e Interna- cionais observa-se que $100 \%$ dos títulos estão indexados na Base de Literatura Latino Americana de Ciências da Saúde (LILACS) fato esperado uma vez que a seleção das revistas, para esta investigação, partiu da consulta inicial nesta fonte. Por outro lado, verificou-se que 9 (39\%) títulos constam apenas dessa fonte enquanto os demais 14 títulos estão representados em outras fontes internacionais: EMBASE (Excerpta Médica), MEDLINE (Index Medicus), Biological Abstracts (BAst.), Chemical Abstracts (CAB), Tropical Diseases Bulletin (CAB), Periódica, Free Medical Journal. 
A presença de 10 títulos na Base SciELO é interessante, na medida em que se observa que desses, 8 constam também de uma ou mais das fontes acima mencionadas. Por outro lado, 2 títulos, além da LILACS, estão indexados somente no SciELO (Revista Brasileira de Cirurgia Cardiovascular e Revista Brasileira de Ginecologia e Obstetrícia). No entanto, 4 revistas que estão indexadas na EMBASE e uma delas também no Biological Abstracts ainda não foram contempladas na seleção das revistas incorporadas ao SciELO (Brazilian Journal of Urology, GED, Revista Brasileira de Anestesiologia e Revista Brasileira de Ortopedia). Observa-se, contudo, que 9 (39\%) constam apenas da base LILACS. As 10 revistas atualmente no SciELO deverão ser conceituadas pela CAPES como nível A, segundo critérios de Avaliação/Qualis Nacional diulgados na palestra "Critérios CAPES para publicações científicas" ministrada pelo Prof. Dr. Jair de Jesus Mari, no dia 23 de outubro de 2002, na UNIFESP-EPM.

\section{COMENTÁRIOS E CONCLUSÃO}

O número de artigos cirúrgicos publicados nas revistas brasileiras é pouco representativo em relação aos não cirúrgicos.

A identificação da produção científica na área de cirurgia segundo o critério de seleção, a partir de conceituada base de indexação, só foi alcançada por meio da análise de conteúdo, realizada por profissionais da área médica, uma vez que os títulos não permitiram atingir este objetivo.

A criteriosa análise identificou um número reduzido de revistas (4\%) que publicaram $50 \%$ ou mais de artigos cirúrgicos, dedicando acima de $40 \%$ das páginas das publicações do triênio, nos quais predominam os artigos originais segundo o aspecto de estudos em seres humanos.

Os artigos cirúrgicos resultantes de pesquisa em animais de experimentação são representados com média acima de $10 \%$ em apenas seis revistas (Arquivos de Gastroenterologia, Revista da Associação Médica Brasileira, Revista do Colégio Brasileiro de Cirurgiões, Revista Brasileira de Mastologia, Jornal de Pneumologia e Acta Cirúrgica Brasileira).

As 23 revistas são consideradas consolidadas quanto ao período de existência e têm a credibilidade garantida pela periodicidade, que é mantida por instituições representativas da comunidade científica. É louvável o esforço que os editores das revistas brasileiras estão fazendo para colocar no domínio público a produção científica do país. A tiragem das publicações editadas com periodicidade regular, quase exclusivamente com recursos não governamentais, é um dos desafios enfrentados para atingir o nível de qualidade. A avaliação da qualidade é feita constantemente por consultores, que se pautam em critérios para elaborar o parecer sobre a inclusão ou não das revistas em bases de dados, ou mesmo pareceres que implicam na divulgação do "ranking" de revistas recomendadas. Os conceitos atribuídos, sem considerar objetivos diferenciados e características de cada uma das áreas do conhecimento, é outro desafio constante.

As análises baseadas na visibilidade da produção científica brasileira nas fontes secundárias estrangeiras, embora de grande repercussão, não foram suficientes para facilitar o acesso ao texto completo e não ofereciam os recursos adequados para os estudos bibliométricos e cienciométricos tão divulgados e discutidos pelo ISI - Institute of Scientific Information. Reconhecendo a necessidade de ter indicadores para a avaliação da produção científica da América Latina, a BIREME enfrentou o desafio e apresenta excelentes resultados com o SciELO - Scientific Eletronic Library Online, onde já incorporou até a presente data 91 periódicos, dos quais 32 são da área de ciências da saúde. Entre esses, estão 10 (44\%) títulos que fazem parte desta investigação.

Os resultados deixam claro que a produção científica publicada no Brasil está cada dia mais visível e acessível à comunidade. Este fato certamente influirá no conceito atribuído às revistas por órgãos responsáveis pela avaliação desta produção, como é o caso da CAPES. Isso pode ser considerado quando se procura entender os parâmetros utilizados pelos responsáveis pelas avaliações.

As avaliações das diferentes áreas da ciência, das instituições, dos pesquisadores e dos países vêm sendo realizadas por especialistas que explicitam os parâmetros utilizados para comparação e atribuição de fatores de impacto considerando-se o contexto sócio-econômico do periódico analisado e o "out-put" real em relação ao "in-put" disponível.

Estudos mais profundos são as metas das próximas etapas desta linha de investigação.

\section{REFERÊNCIAS}

1. Población DA, Goldenberg S. Acta Cirúrgica Brasileira: visibilidade e acessibilidade da produção científica na área de cirurgia experimental. Acta Cir Bras [periódico online] 2001 [Acesso em 18/9/2002];16(3):115-21 [11 telas]. Disponível em: URL: http://www.scielo.br/acb

2. Meneghini R. Avaliação da produção científica e o Projeto SciELO. Ciênc Inf. 1998;27(2):219-20.

3. Price SD. Little science, big science. New York: Columbia University Press; 1963.

4. Garvey WD. Communication: the essence of science. Oxford: Pergamon Press; 1979.

5. Meadows AJ. A comunicação científica. Brasília (DF): Briquet de Lemos/Livros; 1999. 
6. Moravcsick MJ. Como evaluar la ciencia y a los científicos? Rev Esp Doc Cient. 1989; 12(3):313-25.

7. Garfield E. Publication and national research policies: quantitative analysis of the scientific literature and its implications for science policymaking. Presented at International Seminar on the Challenges of the Information Era: agents and users. São Paulo, 18th to 20 th October 1994.

8. Spinak E. Las analisis cuantitativos de la literatura científica y su validez para juzgar la producción latinoamericana. Bol Oficina Sanit Panam 1996;120(2):139-47.

9. Lopez Piñero JM, Terrada ML. Los indicadores bibliométricos y la evaluación de la actividad médica científica (III). Los indicadores de producción, circulación y dispersión; consumo de la información y repercusión. Med Clin 1992;98:142-8.

10. Lopez-Cózar ED. Evaluación del grado de ajuste de las revistas españolas de ciencias de la saud a las normas internacionales de presentación da publicaciones periódicas. Rev Esp Salud Publica 1997a;71:531-46.

11. Lopez-Cózar ED. Incidencia de la normalización de las revistas científicas en la transferencia y evaluación de la información científica. Rev Neurol 1997b;25(148):1942-6.

12. Macias-Chapula C. O papel da informetria e da cienciometria e sua perspectiva nacional e internacional. Ciênc Inf Brasília 1998;27(2):134-40.

13. International Committee of Medical Journal Editors. Declariones del Comité Internacional de Directores de Revistas Médicas anexas a las normas de Vancouver. Rev Panam Salud Publica [periódico online] 1998 [Acesso em 7/11/ 2001];3(4):257-61 [8 telas]. Disponível em URL: http:// www.scielosp.org

14. Mueller SPM. O impacto das tecnologias da informação na geração do artigo científico: tópicos para estudo. Ciênc Inf Brasília 1994;23:309-17.

15. Miranda DB, Pereira MNF. O periódico científico como veículo de comunicação: uma revisão de literatura. Ciênc Inf Brasília 1996;25:375-82.

16. Garfield E. A retrospective and prospective view of information retrieval and artificial intelligence in the $21 \mathrm{st}$ century. J Am Soc Inf Sc Tech 2001;52(1):18-21.

17. Boor M. The citation impact factor. Another dubious index of journal quality. Am Psychol 1982;2:975-7.

18. Garfield E. Which medical journals have the greastest impact? Ann Intern Med 1986;105:313-20.

19. Garfield E. The impact factor. Current contents 1994; 25(20):3-7.

20. Opthof T. Sense and nonsense about the impact factor. Cardiovasc Res 1997;331-7.

21. Wormell I. Informetria: explorando bases de dados como instrumentos de análise. Ciênc Inf La Habana 1998;27(2): 210-6.

22. Elkis H. Fatores de impacto de publicações psiquiátricas e produtividade científica. Rev Bras Psiquiat [periódico online] 1999 [Acesso em 27/1/2002]; 21(4):231-6 [1 tela]. Disponível em http://www.scielo.br

23. O'Neil J. The significance of an impact factor: implications for the publishing community. Learned publishing 2000;(13):105-9.

24. Vilhena V, Crestana MF. Produção científica: critérios de avaliação de impacto. Rev Assoc Med Bras 2002;48(1):20-1.

25. Kassirer J. Why be a medical editor? J Am Med Assoc 2001;285(17):2253.

26. Costa AFC. Estrutura da produção editorial de periódicos biomédicos brasileiros. [dissertação de mestrado]. IBICT/UFRJ; 1988.
27. Greene LJ. Periódicos científicos brasileiros: problemas e perspectivas. In: $2^{\circ}$ Workshop de Editores Científicos. Petrópolis: 17-19 novembro 1999. Livro de Resumos. p.12.

28. Utizer RD. A syllabus for prospective and newly appointed editors. Disponível em http://www.wame.org/syllabus. (Acesso em 15/2/2002).

29. Perez Gómez MA. papel del editor y de los comites editoriales como guardianes de la calidad de las revistas. Cienc Inf La Habana 1998;29:37-44.

30. Goldenberg S. Núcleo de comunicação científica em cirurgia [NCCC] e núcleo dos amigos da cirurgia experimental [NACE] da SOBRADPEC [editorial]. Acta Cir Bras [períodico online] 2002;17(2):87-8[3 telas]. Disponível em URL: http:// www.scielo.br/acb

31. Azevedo JLMC, Población DA, Goldenberg S. Descritores unitermos nos artigos científicos. Acta Cir Bras 1990;5(2): 35-54.

32. Goldenberg S. A desigualdade de critérios nas referências bibliográficas [editorial]. Acta Cir Bras 1993;8(4):143-4.

33. Goldenberg S, Población DA, Gomes PO, Soares AL, Ferreira JR, Kafejian AP, Braga MER. Editoração de revistas científicas: análise das instruções aos autores de 19 revistas brasileiras. Acta Cir Bras 1995;10(2):55-60.

34. Población DA, Goldenberg S, Ferreira JR, Soares AL. O periódico como produto: da criação do autor à inovação do editor. In: 7. Encontro de Editores Científicos. Caxambú, 7-10 out. 1998. Caxambú, ABEC Livro de Resumos, p.7.

35. Goldenberg S. Aspectos éticos da pesquisa em seres humanos contidos nas instruções aos autores em revistas científicas brasileiras. Rev Assoc Med Bras 1999;45(4):289.

36. Goldenberg S. Considerações éticas a respeito da publicação do trabalho científico. In: Petroianu A. Ética, moral e deontologia médicas. Rio de Janeiro: Guanabara Koogan; 2000. p.205-6.

37. Goldenberg S. Normalizar é salutar [editorial]. Acta Cir Bras 2000;15(2):79.

38. Población DA, Goldenberg S, Gomes PO, Soares AL, Ferreira JR, Kafejian AP, Braga MER. A comunicação científica por meios eletrônicos: o caso das publicações médicas. Acta Cir Bras 1996;11(1):36-8.

39. Silva LAG. El impacto de las publicaciones eletrónicas en la comunicación científicas brasileñas. Cienc Inf La Habana 1998;29(1):61-7.

40. Barreto AA. Mudança estrutural no fluxo do conhecimento: a comunicação eletrônica. Cien Inf Brasília 1998;27(2):122-7.

41. Packer AL. SciELO: uma metodologia para publicação eletrônica. Cienc Inf Brasília 1998; 27(2):109-21.

42. Licea de Arenas J, Valles J, Morales V. Indicadores de calidad de las revistas científicas. Ciênc Inf La Habana 1999;30(3):3-14.

43. Castro RCF, Ferreira MCG, Vidili AL. Periódicos latinoamericanos: avaliação das características formais e sua relação com a qualidade científica. Cienc Inf Brasília 1996; 25(3): 357-67.

44. Viesca R. Mendez A. Métodos para la valoración de las revistas científicas. Rev Esp Doc Cient 1979;2:357-63.

45. Council of Biology Editors. Manual de estilo CBE. Guia para autores, editores y revisores en el campo de la medicina y la biologia. Barcelona: Salvat; 1987.

46. Huth E. Medical Style \& Format: an international manual for authors, editors and publishers. Filadelfia: ISI; 1987.

47. International Committee of Medical Journal Editors. Uniform requirements for manuscripts submitted to biomedical journals. Filadelfia, Octuber 2001. Disponível em http://www.icmje.org. (Acesso em 18/08/2002). 
Agradecimentos pela colaboração na coleta de dados

Aos alunos da pós-graduação e graduação da UNIFESP-EPM e outras entidades: Charles Alfabet, Cláudio Sérgio Salim, Dinamar José Zanchet, Heliane Campanatti Ostiz, Marcos de Souza Abrahão, Mônica Cecília Bochetti Manna, Marcelo Eduardo Pedrosa, Vanessa Contato Lopes Resende e Alexandre Gavira Marques.

Aos funcionários da Biblioteca Central da UNIFESP-EPM:

Maria Elisa Rangel Braga, Andréa Cristina F. Carmo, Clarice Vidal da Silva, Isabel Bueno, Santos Menezes, Márcia Regina da Sílvia, Reinaldo Ramos de Carvalho e Teresa Avalos.

Conflito de interesse: nenhum

Endereço para correspondência:

Fonte de financiamento: nenhuma

Profa. Dinah Aguiar Población

Rua Jorge Rizzo, 217/133

05424-060 São Paulo - SP

Tel/Fax (11) 3815-7309

Data do recebimento: $11 / 07 / 2002$

Data da revisão: 20/08/2002

Data da aprovação: 25/09/2002 